\title{
Impedance Characteristics of Log Periodic Antenna
}

\author{
B. I. Neelgar \\ GMR Institute of Technology \\ GMR Nagar, \\ Rajam-532127, Srikakulum (Dist) \\ A. P. (India)
}

\author{
Prof. GSN Raju \\ Principal \\ A.U. College of Engineering \\ Andhra University, \\ Visakhapatnam-530003 A.P. (India)
}

\begin{abstract}
Log Periodic array Antenna is one of the most important and commercially used antennas for T.V. reception. It is used in VHF and UHF bands. Although the analysis of this antenna is reported in literature, the data of self impedance \& mutual impedance is not fully available. But, this data is useful for the optimal design of the antenna. In view of this the array above is considered and the analysis is carried out in the present work. The computed data for Self-Impedance as a function of lengths of elements and frequency and the Mutual Impedance as a function of spacings of elements is presented.
\end{abstract}

Keywords

Log-Periodic, Self Impedance, Mutual Impedance, Hertzian Dipole

\section{INTRODUCTION}

Log Periodic antenna introduced by DuHamel and Isbell [1] is frequency independent antenna which has a structural geometry such that its impedance is periodic with logarithm of the frequency. Fig. 1 shows a schematic structure of a $\log$ periodic antenna.

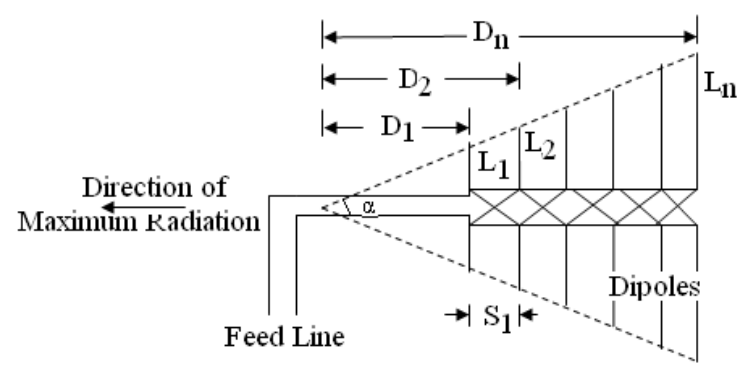

Figure. 1 Log Periodic Antenna Array

The antenna was designed and introduced by DuHamel. The antenna consists of a metal strip whose edges are specified by the angle $\alpha / 2$. The length from the origin to any point on the structure is specified including a distance characteristic as [2]

$$
\theta=\theta_{0} \sin \left[b \ln \left(\frac{r}{r_{0}}\right)\right]
$$

From equation (1) it can be seen that the values of $\theta$ are separated whenever the logarithm of the radial frequency $\ln (\omega)=\ln (2 \pi \mathrm{f})$ differs by $2 \pi / \mathrm{b}$. The performance the antenna is periodic as function of logarithm of frequency and hence the name is log-periodic.

The array of log-periodic antenna consists of a sequence of sideby-side parallel linear dipoles forming a coplanar array as shown in Figure 1. The lengths, spacings and diameters increase logarithmically as defined by the geometric ratio $\tau$. That is, [3]

$$
\tau=\frac{D_{n}}{D_{n-1}}=\frac{L_{n}}{L_{n-1}}, \quad n=1,2,3, \ldots
$$

The spacing factor $\sigma$ is defined as

$$
\begin{aligned}
& \sigma=\frac{S_{n}}{2 L_{n}}=\frac{S_{n}}{S_{n-1}} \\
& S_{n}=D_{n-1}-D_{n} \\
& \text { where } \alpha=\text { Wedge angle }=2 \tan ^{-1}\left(\frac{1-\tau}{4 \sigma}\right)
\end{aligned}
$$

In Yagi_Uda antenna only one element of the array is directly energized by the feed line, while others operate in parasitic mode whereas in log periodic antenna array all the elements are connected to the feed line. Two methods are used to connect feed line wherein in the first type the currents in the elements have same phase relationship as the terminal phases. This produces an endfire beam in the direction of longer elements. In the second method the feed is crisscrossed or transposed between the adjacent elements wherein a $180^{\circ}$ phase is added to the terminal of each element. Since the phase between the adjacent closely placed short elements is almost in opposition, very little energy is radiated by them and their interference effects are negligible. But at the same time the longer and larger spaced elements radiate. The mechanical phase reversal between these elements produces a phase progression so that energy is radiated endfire in the direction of shorter elements. The lowest cutoff frequency occurs approximately when the longest element is $\lambda / 2$. Once the length of longest element is known then the lengths of other elements can be calculated by the relation shown in (2) with the knowledge of $\tau$. The values of $\tau$ and $\sigma$ are obtained from Isbell curves. [4]. With $\sigma$ the spacings and diameters are obtained with a given frequency band. 


\section{ANALYSIS OF ARRAY FOR SELF IMPEDANCE}

The radiation characteristics of an antenna in the presence of a lossy ground depend substantially on the infinite ground conductivity and in homogeneity [5-7]. The problem was conventionally simplified on the basis of a Hertzian dipole with specified current moment for very low frequency range . But, for higher frequency a finite length antenna should be considered.

In the present analysis, the array is considered to be symmetric and the element half length is not greater than the limit of $5 \lambda / 8$. The analysis is carried out by King and Wu's three term assumption for the current [8-10]. Thus, for a single centre-fed dipole in free space [11]

$$
\begin{aligned}
& \qquad \begin{array}{l}
I(h)=\frac{j V}{60 \Gamma_{d A} \cos \beta l}[\sin \beta(l-|h|)+A+B] \quad \text { for } \quad \beta l \neq \frac{\pi}{2} \\
\text { where } \quad A=X_{X}(\cos \beta h-\cos \beta l) \\
B=X_{Y}\left(\cos \frac{\beta h}{2}-\cos \frac{\beta l}{2}\right)
\end{array}
\end{aligned}
$$

or

$$
\begin{aligned}
& I(h)=\frac{-j V}{60 \Gamma_{d A}}[\sin \beta|h|-1+C+D] \quad \text { for } \quad \beta l=\frac{\pi}{2} \\
& \text { where }
\end{aligned}
$$

$$
D=X_{Y}^{\prime}\left(\cos \frac{\beta h}{2}-\cos \frac{\pi}{4}\right)
$$

In the above equations (4) and (5), $\mathrm{V}$ is the applied voltage, $h$ is the distance along the dipole axis measured from the feeding point, $l$ is the half-length of the dipole, $a$ is the radius of dipole, $\beta=2 \pi / \lambda$ and the other symbols are as defined below[11].

$$
\begin{aligned}
& \Gamma_{d A}=2 C 1 \overline{C_{c}}(\beta l)-2 \cos \beta l \overline{C_{c}}(2 \beta l) \\
& -2 \cot \beta l C 1 C_{s}(\beta l)+E X P . C_{s}(2 \beta l) \\
& \begin{array}{ll}
\text { where } & C 1=1+\cos \beta l \\
& E X P=(\cot \beta l \cos \beta l-\sin \beta l)
\end{array}
\end{aligned}
$$

\section{For $\beta 1 \geq \pi / 2$}

$$
\begin{aligned}
& \Gamma_{d A}=\overline{C_{c}}(0.5 \pi)+C 2 \overline{C_{c}}(E)-\cos 2 \beta l \overline{C_{c}}(2 E) \\
& +\sin 2 \beta l\left[\overline{C_{c}}(\beta l)-\overline{C_{c}}(2 \beta l)\right]-C 2 C_{s}(\beta l) \\
& +\cos 2 \beta l C_{s}(2 \beta l)+\sin 2 \beta l\left[C_{s}(E)-C_{s}(2 E)\right] \\
& \text { where } \quad C 2=1+\cos 2 \beta l
\end{aligned}
$$

when $\boldsymbol{\beta I}=\boldsymbol{\pi} / \mathbf{2}$

$$
\Gamma_{d A}\left(\beta l=\frac{\pi}{2}\right)=2 \overline{C_{c}}(0.5 \pi)-C_{s}(\pi)
$$

The complex functions $\mathrm{X}_{\mathrm{X}}, \mathrm{X}_{\mathrm{Y}}, \mathrm{X}_{\mathrm{X}}^{\prime}$ and $\mathrm{X}_{\mathrm{Y}}^{\prime}$ in equations (4) and (5) are expressed as

$$
\begin{aligned}
& X_{X}=Z^{-1}\left(\Gamma_{d B} \Gamma_{V}-j \Gamma_{d C} \Gamma_{A}\right) \\
& X_{Y}=-j Z^{-1}\left[\begin{array}{l}
\Gamma_{d C}\left(\Gamma_{d E} \cos \beta l-\Gamma_{U}\right) \\
+\Gamma_{d F} \Gamma_{V}
\end{array}\right] \\
& X^{\prime}{ }_{X}=\frac{\Gamma_{d B} \Gamma_{d A}}{\Gamma_{d B} \Gamma_{V}-j \Gamma_{d C} \Gamma_{A}} \\
& X_{Y}^{\prime}=\frac{j \Gamma_{d C} \Gamma_{d E}}{\Gamma_{d B} \Gamma_{V}-j \Gamma_{d C} \Gamma_{A}} \\
& \text { where } \quad Z=\Gamma_{d B}\left(\Gamma_{d E} \cos \beta l-\Gamma_{U}\right)+j \Gamma_{d F} \Gamma_{A}
\end{aligned}
$$

The other symbols in the above equations are as follows:

$$
\begin{aligned}
\Gamma_{d E}= & \frac{2}{C 3} \bar{C}_{c}(\beta l)-\frac{\cos \beta l}{C 3} \bar{C}_{c}(2 \beta l) \\
& -\frac{2 \cos \beta l}{C 3} \bar{C}(\beta l)+\frac{\cos \beta l}{C 3} \bar{C}(2 \beta l) \\
& -\frac{\sin \beta l}{C 3} C_{s}(2 \beta l) \\
\Gamma_{d F}= & \frac{1}{C 4}\left[\begin{array}{l}
-2 S_{c}(\beta l)+\cos \beta l S_{c}(2 \beta l) \\
+2 \cos \beta l S(\beta l)-\cos \beta l S(2 \beta l) \\
+\sin \beta l S_{s}(2 \beta l)
\end{array}\right. \\
\Gamma_{d C}= & \frac{1}{C 4}\left\{\begin{array}{l}
-2 \sin \beta l S_{c}(\beta l)+2 \cos \beta l S_{s}(\beta l) \\
-\sin 2 \beta l\left[S_{c}(\beta l)-S_{c}(2 \beta l)\right] \\
+C 2 S_{s}(\beta l)-\cos 2 \beta l S_{s}(2 \beta l)
\end{array}\right\} \\
\Gamma_{d B}= & \frac{1}{C 4}\left[\begin{array}{l}
\left.G_{1}+j H_{1}\right]
\end{array}\right.
\end{aligned}
$$

With

$$
\begin{aligned}
G_{1}= & 2 \sinh ^{-1}\left(\frac{l}{a}\right)-C(P)-C(Q) \\
& -\sinh ^{-1}\left(\frac{2 l}{a}\right) \cos \frac{\beta l}{2}+\frac{1}{2} \cos \frac{\beta l}{2} C(R) \\
& -\frac{1}{2} \sin \frac{\beta l}{2} S(R)+\cos \frac{\beta l}{2} \bar{C}(2 \beta l) \\
& +\frac{1}{2} \cos \frac{\beta l}{2} C(T)+\frac{1}{2} \sin \frac{\beta l}{2} S(T) \\
& -2 \cos \frac{\beta l}{2} \bar{C}(\beta l)
\end{aligned}
$$




$$
\begin{aligned}
& H_{1}=-S(P)-S(Q)+\frac{1}{2} \sin \frac{\beta l}{2} C(R) \\
&+\frac{1}{2} \cos \frac{\beta l}{2} S(R)+\frac{1}{2} \cos \frac{\beta l}{2} S(T) \\
&-\frac{1}{2} \sin \frac{\beta l}{2} C(T)+2 \cos \frac{\beta l}{2} S(\beta l) \\
&-\cos \frac{\beta l}{2} S(2 \beta l) \\
& \Gamma_{V}= G_{2}+j H_{2} \\
& G_{2}=-\sin 2 \beta l\left[\bar{C}_{c}(\beta l)-\overline{C_{c}}(2 \beta l)\right] \\
&+\cos 2 \beta l\left[C_{s}(\beta l)-C_{s}(2 \beta l)\right]+C_{s}(\beta l) \\
& H_{2}=\sin 2 \beta l\left[S_{c}(\beta l)-S_{c}(2 \beta l)\right] \\
&-\cos 2 \beta l\left[S_{s}(\beta l)-S_{s}(2 \beta l)\right]-S_{s}(\beta l) \\
& \Gamma_{U}=\cos \beta l\left[\overline{C_{c}}(2 \beta l)-\overline{C_{c}}(2 \beta l)\right] \\
& \quad+\sin k h C_{s}(2 \beta l) \\
&+j\left\{\begin{array}{l}
\cos \beta l\left[S(2 \beta l)-S_{c}(2 \beta l)\right] \\
-\sin \beta l S_{s}(2 \beta l)
\end{array}\right\} \\
& \Gamma_{A}=G_{3}+j H_{3}
\end{aligned}
$$$$
\text { With } G_{3}=\cos \frac{\beta l}{2}\left[\begin{array}{l}
\sinh ^{-1}\left(\frac{l}{a}\right)-\frac{1}{2} C(R) \\
-\frac{1}{2} C(T)-\bar{C}(2 \beta l)
\end{array}\right]
$$$$
+\frac{1}{2} \sin \frac{\beta l}{2}[S(R)-S(T)]
$$$$
H_{3}=\cos \frac{\beta l}{2}\left[S(2 \beta l)-\frac{1}{2} S(R)-\frac{1}{2} S(T)\right]
$$$$
+\frac{1}{2} \sin \frac{\beta l}{2}[C(T)-C(R)]
$$

For a set of values for $\boldsymbol{a}$ and $\boldsymbol{l}$ all the functions shown above can be calculated. Thus from equations (4) and (5) the self impedance of the dipole is computed as

$$
Z_{s}=\frac{-j 60 \Gamma_{d A} \cos \beta l}{\sin \beta l+X_{x} C 3+X_{Y} C 4}
$$

$$
\begin{array}{r}
Z_{s}=\frac{j 60 \Gamma_{d A}}{-1+X_{X}^{\prime}-(1-\sqrt{2} / 2) X_{Y}^{\prime}} \\
\text { for } \quad \beta l=\frac{\pi}{2}
\end{array}
$$

In the equations from (4) through (28) the various symbols used are defined as follows. The generalized sine and cosine integrals are defined as:

$$
\begin{aligned}
& C_{s}(\beta l)=C_{s}(\beta a, \beta l) \\
& C_{s}(2 \beta l)=C_{s}(\beta a, 2 \beta l) \\
& C_{s}(0.5 \pi)=C_{s}(\beta a, \pi / 2) \\
& C_{s}(E)=C_{s}(\beta a, \beta l-\pi / 2) \\
& C_{s}(2 E)=C_{s}(\beta a, 2 \beta l-\pi / 2) \\
& C_{s}(\pi)=C_{s}(\beta a, \pi) \\
& C_{s}(E)=C_{s}(\beta a, \beta l-\pi / 2) \\
& C_{s}(2 E)=C_{s}(\beta a, 2 \beta l-\pi / 2) \\
& C_{s}(P)=C_{s}\left(\beta a \frac{\sqrt{3}}{2}, \frac{\beta l}{2}\right) \\
& C_{s}(Q)=C_{s}\left(\beta a \frac{\sqrt{3}}{2}, \frac{3 \beta l}{2}\right) \\
& C_{s}(R)=C_{s}\left(\beta a \frac{\sqrt{3}}{2}, 3 \beta l\right) \\
& C_{s}(T)=C_{s}\left(\beta a \frac{\sqrt{3}}{2}, \beta l\right)
\end{aligned}
$$

The expressions hold good for other integrals shown in equations (4) through (28). These generalized integrals are given in the reference [11] and Appendix. The other symbols used are defined as follows:

$$
\begin{aligned}
& C 1=(1+\cos \beta l) \\
& C 2=(1+\cos 2 \beta l) \\
& C 3=(1-\cos \beta l) \\
& C 4=\left(1-\cos \frac{\beta l}{2}\right)
\end{aligned}
$$

$$
\text { for } \quad \beta l \neq \frac{\pi}{2}
$$




\section{ANALYSIS OF ARRAY FOR MUTUAL IMPEDANCE}

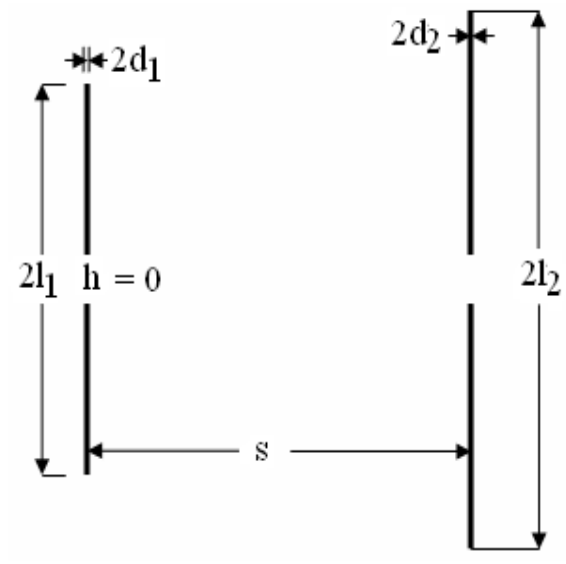

Figure. 2 Two non-identical element array

The open circuit mutual impedance between two parallel dipoles of half-lengths $l_{1}$ and $l_{2}$ spaced a distance $s$ apart as shown in Figure. 2 is calculated by

$$
Z_{m}=\frac{-1}{I_{1}(0) I_{2}(0)} \int_{-l 2}^{l 1} E_{s 1}(h) I_{2}(h) d h
$$

where $I_{1}(0)$ and $I_{2}(0)$ are the input currents of No.1 and No.2 antennas respectively. The $\boldsymbol{h}$ component of electric field $\mathrm{E}_{\mathrm{s} 1}(\mathrm{~h})$ produced at No. 2 antenna by the current on No. 1 antenna is given by [11]

$$
E_{h 1}=\frac{-j 30}{\beta}\left(\beta^{2}+\frac{\partial^{2}}{\partial h^{2}}\right)_{-l 1}^{l 1} \int_{1}^{1}\left(h^{\prime}\right) K\left(h, h^{\prime}\right) d h^{\prime}
$$

where $K\left(h, h^{\prime}\right)=\frac{e^{-j \beta R}}{R}$

$$
R=\left[\left(h-h^{\prime}\right)^{2}+s^{2}\right]^{1 / 2}
$$

Substituting equations (35) into (34) and using (4) and (5) for $\mathrm{I}(\mathrm{h}$ ') and $\mathrm{I}(\mathrm{h})$ the expression for mutual impedance can be obtained as

a) for $\beta \mathrm{l}_{1} \neq \pi / 2$ and $\beta \mathrm{l}_{2} \neq \pi / 2$,

$$
Z_{m}=\frac{-j 30\left\{-\left[\begin{array}{l}
\beta M_{a}+2\left(\cos \beta l_{1}\right) M_{b} \\
+\frac{1}{2} X_{Y 1} \sin \left(\beta l_{1} / 2\right)
\end{array}\right]\right\}}{M_{c}}
$$

where

$$
\begin{array}{r}
Y_{a}=\sin \beta l_{1}+X_{X 1}\left(1-\cos \beta l_{1}\right) \\
+X_{Y 1}\left(1-\cos \frac{\beta l_{1}}{2}\right) \\
Y_{b}=\sin \beta l_{2}+X_{X 2}\left(1-\cos \beta l_{2}\right) \\
+X_{Y 2}\left(1-\cos \frac{\beta l_{2}}{2}\right)
\end{array}
$$

$$
M_{a}=\int_{-l 2}^{l 2}\left[\begin{array}{l}
\sin \beta\left(l_{2}-|h|\right)+X_{X 2} C 5 \\
+X_{Y 2} C 6
\end{array}\right]
$$$$
\times \int_{0}^{l 1}\left(\begin{array}{l}
X_{X 1} \cos \beta l_{1}-\frac{3}{4} X_{Y 1} \cos \frac{\beta h^{\prime}}{2} \\
+X_{Y 1} \cos \frac{\beta l_{1}}{2}
\end{array}\right)
$$

$K K d h^{\prime} d h$

$$
\begin{gathered}
M_{b}=\int_{-l 2}^{l 2} K(h, 0)\left[\begin{array}{l}
\sin \beta\left(l_{2}-|h|\right) \\
+X_{X 2} C 5 \\
+X_{Y 2} C 6
\end{array}\right] d h \\
M_{c}=\int_{-l 2}^{l 2} K K\left[\begin{array}{l}
\sin \beta\left(l_{2}-|h|\right) \\
+X_{X 2} C 5+ \\
X_{Y 2} C 6
\end{array}\right] d h
\end{gathered}
$$

b) for $\beta \mathrm{I}_{1}=\pi / 2$ and $\beta \mathrm{I}_{2} \neq \pi / 2$,

$$
Z_{m}=\frac{j 30\left\{\begin{array}{l}
\beta M_{d}+2 M_{b}+ \\
\left.\left[X_{X 1}^{\prime}-\frac{1}{2} X_{Y 1}^{\prime} \sin (\pi / 4)\right]\right\} \\
M_{c}
\end{array} Y_{c} Y_{b}\right.}{Y^{\prime}}
$$


where

$$
\begin{aligned}
Y_{c} & =-1+X_{X 1}^{\prime}-X_{Y 1}^{\prime}\left(1-\cos \frac{\pi}{4}\right) \\
M_{d} & =\int_{-l 2}^{l 2}\left[\begin{array}{l}
\left.\sin \beta\left(l_{2}-|h|\right)+X_{X 2} C 5+\right] \\
X_{Y 2} C 6
\end{array}\right] K K d h^{\prime} d h \\
& \times \int_{0}^{l 1}\left(\begin{array}{l}
-1+\frac{3}{4} X_{X 1}^{\prime} \cos \frac{k h^{\prime}}{2} \\
+X_{Y 1}^{\prime} \cos \frac{\pi}{4}
\end{array}\right)
\end{aligned}
$$$$
\text { c) for } \beta \mathrm{l}_{1}=\beta \mathrm{l}_{2}=\pi / 2 \text {, }
$$$$
Z_{m}=\frac{j 30\left\{\begin{array}{l}
\beta M_{e}+2 M_{f} \\
+\left[X_{X 1}^{\prime}-\frac{1}{2} X_{Y 1}^{\prime} \sin (\pi / 4)\right] M_{g}
\end{array}\right\}}{Y_{c} Y_{d}}
$$

where

$$
Y_{d}=-1+X_{X 2}^{\prime}-X_{Y 2}^{\prime}\left(1-\cos \frac{\pi}{4}\right)
$$

(which will be identical to $M_{c}$ if $d_{2}=d_{1}$ )

$$
\begin{aligned}
M_{e} & =\int_{-l 2}^{l 2}\left[\begin{array}{l}
\sin \beta|h|-1+X_{X 2}^{\prime} \cos \beta h \\
-X_{Y 2}^{\prime} C 7
\end{array}\right] \\
& \times \int_{0}^{l 1}\left(-1+\frac{3}{4} X_{X 1}^{\prime} \cos \frac{k h^{\prime}}{2}+X_{Y 1}^{\prime} \cos \frac{\pi}{4}\right)
\end{aligned}
$$

$K K d h^{\prime} d h$

$$
\begin{aligned}
& M_{f}=\int_{-l 2}^{l 2} K(h, 0)\left[\begin{array}{l}
\sin \beta|h|-1 \\
+X_{X 2}^{\prime} \cos \beta h \\
-X_{Y 2}^{\prime} C 7
\end{array}\right] d h \\
& M_{g}=\int_{-l 2}^{l 2} K K\left[\begin{array}{l}
\sin \beta|h|-1 \\
+X_{X 2}^{\prime} \cos \beta h-X_{Y 2}^{\prime} C 7
\end{array}\right] d h
\end{aligned}
$$

$$
\begin{aligned}
& C 5=\cos \beta h-\cos \beta l_{2} \\
& C 6=\cos \frac{\beta h}{2}-\cos \frac{\beta l_{2}}{2} \\
& C 7=\cos \frac{\beta h}{2}-\cos \frac{\pi}{4} \\
& K K=K\left(h, h^{\prime}\right)+K\left(h,-h^{\prime}\right)
\end{aligned}
$$

Since $X_{X}, X_{Y}, X_{X}^{\prime}$ and $X_{Y}^{\prime}$ are given in equations (9) to (12), the integrals $M a$ through $M g$ can all be computed numerically, once the values for $d_{1}, d_{2}, l_{1}, l_{2}$ and $s$ are specified. Hence the mutual impedance in (33), (39) or (42) can also be computed. All the equations from (4) to (46) are given in reference [11]. Also, when $\mathrm{X}_{X 1}=X_{X 2}=X_{Y 1}=X_{Y 2}=0$, equation (33) reduces to familiar expression for the case of simple sinusoidal current distribution. In such case the mutual impedance is calculated as [12]

$Z_{m}=-30 \int_{0}^{l_{2}}\left(\begin{array}{l}\frac{-j e^{-j \beta r_{1}}}{r_{1}}-\frac{j e^{-j \beta r_{2}}}{r_{2}} \\ +\frac{2 j \cos k h_{1} e^{-j \beta r_{0}}}{r_{0}}\end{array}\right)$

$$
\sin \beta\left(l_{2}-h\right) d h
$$

$$
\begin{aligned}
r_{0} & =\sqrt{s^{2}+h^{2}} \\
\text { where } \quad r_{1} & =\sqrt{s^{2}+\left(l_{1}-h\right)^{2}} \\
r_{2} & =\sqrt{s^{2}+\left(l_{1}+h\right)^{2}}
\end{aligned}
$$

\section{RESULTS}

In order to validate the impedances, an array of LogPeriodic antenna is considered with Gain $=9 \mathbf{d B}$. The frequency range was selected as $54 \mathrm{MHz}$ to $600 \mathrm{MHz}$ From Isbell curves for $9 \mathrm{~dB}$ gain, $\boldsymbol{\tau}=\mathbf{0 . 8 6 1} \& \boldsymbol{\sigma}=\mathbf{0 . 1 6 2}$. With this data obtained the lengths, spacings and diameters are evaluated. The number of dipole elements is taken as $n=10$. The numerical integrations of the generalized sine and cosine integrals and the impedances were computed using MATLAB. The impedances were computed for frequency of $54 \mathrm{MHz}$ were plotted as shown in the figures [3-6] that follow.

In the above equations the symbols used are defined as: 
The variation of self impedances with lengths of elements for a fixed frequency of $54 \mathrm{MHz}$ is shown in Figure 3 below.

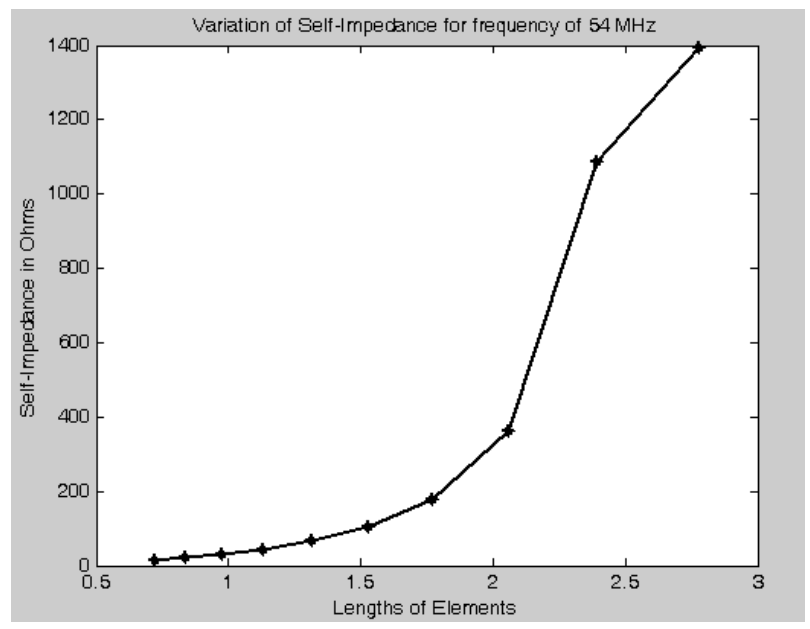

Figure 3 Self Impedance for frequency $=54 \mathrm{MHz}$

The variation of Mutual Impedances with spacings between elements is shown in Figure. 4. Note that $Z_{12}=Z_{21}, Z_{13}=Z_{31}$, $\mathrm{Z}_{23}=\mathrm{Z}_{32} \ldots \ldots, \mathrm{Z}_{910}=\mathrm{Z}_{109}$.

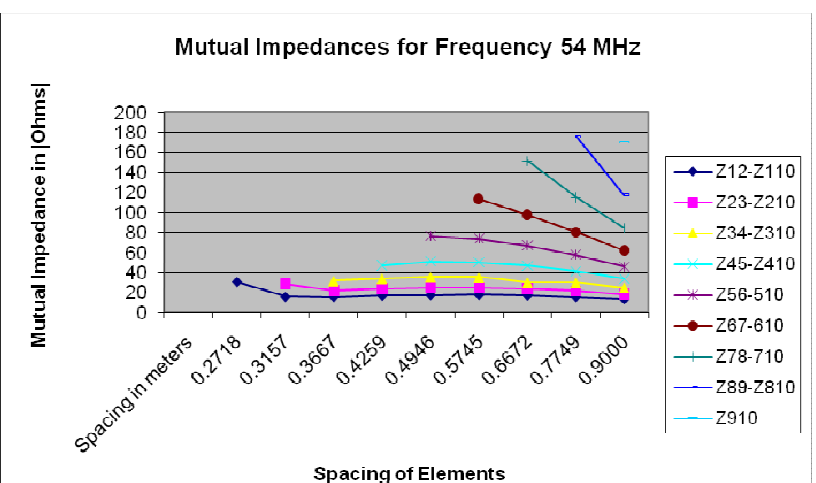

Figure. 4. Variation of Mutual Impedance

Figure 5 illustrates the variation of Self Impedances with different frequencies.

The variation of absolute self impedance with logarithm of frequency for the first three elements is shown in Figure. 6.

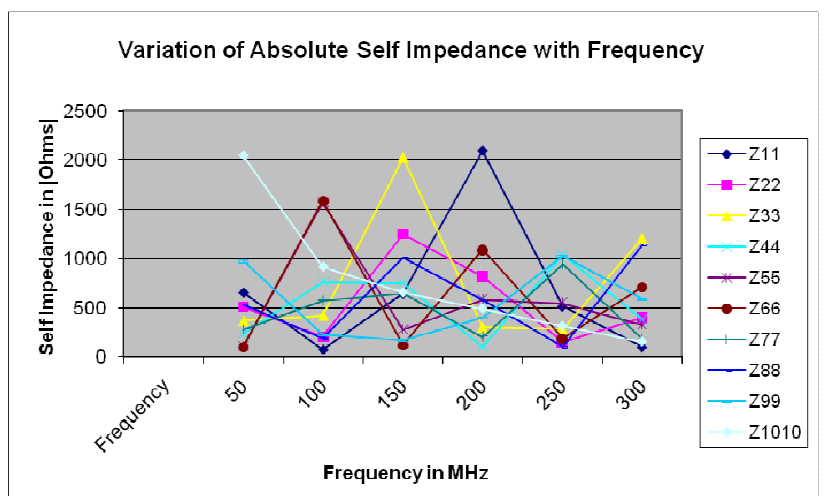

Figure 5 Variation of Self Impedance with Frequency

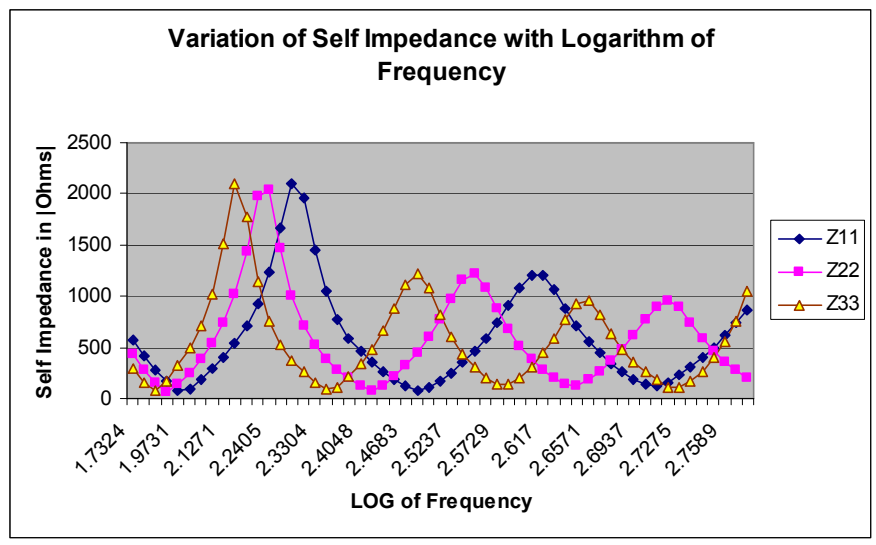

Figure 6. Variation of Self Impedance with Log of Frequency

\section{CONCLUSIONS}

The following conclusions are evident from results presented above.

\subsection{Self Impedance}

- The self impedance is purely dependent on frequency of operation

- As it can be seen from results the self impedance for frequency of $54 \mathrm{MHz}$ is increasing from smallest element to largest element but it may not be true for all frequencies.

- The variation of self impedance with frequency is periodic and hence the name Log-Periodic. 


\subsection{Mutual Impedance}

- For a fixed operating frequency the absolute value of mutual impedance seems to decrease from smallest element to largest element, but it also depends on spacing of elements.

- For a fixed operating frequency the absolute value of mutual impedances depends on length and spacing of the elements between each element.

\section{REFERENCES}

[1] R.H. DuHamel and D. E. Isbell, Broadband Logarithmically Periodic Antenna Structures, 1957 IRE National Convention Record, pt. 1, pp. 119-128

[2] Constantine A. Balanis, Antenna Theory, John Wiley \& Sons, 1997

[3] Dr. G.S.N. Raju, Antennas \& Wave Propagation, Pearson Education, 2005

[4] D. E. Isbell, Log Periodic Dipole Arrays, IRE Transactions, Antennas Propagation., Vol, AP-8, pp. 260267, May 1960

[5] Banos, A., Dipole Radiation in the presence of a conducting half space, Peragamon Press, Oxford England, 1966.

[6] King R. W. P., Theory of Linear Antennas, Harvard University Press, Cambridge, Mass, 1956.

[7] Bhattacharya B.K., Input resistances of horizontal electric and vertical magnetic dipoles over a homogeneous ground, IEEE Transactions, Antennas \& Propagation, Vol. AP-11, No.3 pp. 261-266, May 1963.

[8] King. R.W.P. and T.T. Wu, Currents, Charges near fields of antennas, Radio Sci., Vol. 69D, No. 3, pp 429-446, March, 1965.

[9] Johnson J. H. Wang, The Physical Foundation,Developmental History, and Ultra-wideband Performance of SMM (Spiral-Mode Microstrip) Antennas, IEEE Symposium, Antennas \& Propagation, pp. 586589,July 2005

[10] Y. Mushiake, "A Report on Japanese Development of Antennas: From the Yagi-Uda Antenna to Selfcomplementary Antennas," IEEE Antennas and Prop. Symp. Dig, June 2003.

[11] M. T. Maa, Theory and Applications of Antenna Arrays, John Wiley \& Sons, 1974

[12] Edward C. Jordan, Keith G. Balmin, Electro-Magnetic waves and radiating systems, $\mathrm{PHI}, 2001$

\section{APPENDIX}

The generalized sine and cosine integrals appearing in equations (6) to (28) are expressed as [9]

$$
\begin{aligned}
& S(b, x)=\int_{0}^{x} \frac{\sin \sqrt{y^{2}+b^{2}}}{\sqrt{y^{2}+b^{2}}} d y \\
& C(b, x)=\int_{0}^{x} \frac{1-\cos \sqrt{y^{2}+b^{2}}}{\sqrt{y^{2}+b^{2}}} d y
\end{aligned}
$$

$$
\bar{C}(b, x)=\int_{0}^{x} \frac{\cos \sqrt{y^{2}+b^{2}}}{\sqrt{y^{2}+b^{2}}} d y
$$

$$
S_{s}(b, x)=\int_{0}^{x} \frac{\sin y \sin \sqrt{y^{2}+b^{2}}}{\sqrt{y^{2}+b^{2}}} d y
$$

$$
\begin{aligned}
& S_{c}(b, x)=\int_{0}^{x} \frac{\cos y \sin \sqrt{y^{2}+b^{2}}}{\sqrt{y^{2}+b^{2}}} d y \\
& C_{s}(b, x)=\int_{0}^{x} \frac{\sin y \cos \sqrt{y^{2}+b^{2}}}{\sqrt{y^{2}+b^{2}}} d y
\end{aligned}
$$$$
C_{c}(b, x)=\int_{0}^{x} \frac{(1-\cos y) \cos \sqrt{y^{2}+b^{2}}}{\sqrt{y^{2}+b^{2}}} d y
$$$$
\bar{C}_{c}(b, x)=\int_{0}^{x} \frac{\cos y \cos \sqrt{y^{2}+b^{2}}}{\sqrt{y^{2}+b^{2}}} d y
$$

The numerical integrations are carried out using MATLAB in all the analysis carried out for impedance calculations.

\section{AUTHORS PROFILE}

B. I. Neelgar received his B.E. in Electronics and Communication Engineering from Karnataka University, Dharawad, Karnataka State, India in the year 1984 and M. Tech in Digital Electronics and Advanced Communication from Karnataka Regional Engineering College (NITK), Surathkal, Mangalore University, Karnataka State, India in the year 1995. Presently he is pursuing his Ph.D from Jawaharlal Nehru Technological University (JNTU), Hyderabad. He has an Industry experience of 4.5 years and Teaching experience of 20 years. His areas of interest include Antennas, Antenna Arrays and Communication. 
Dr. G.S.N. Raju received his B.E., M.E. with distinction and first rank from Andhra University and Ph.D. from IIT, Kharagpur. He is Professor of Electronics and Communication Engineering in Andhra University, College of Engineering, Visakhapatnam, India. He is in teaching and research for the last 30 years in Andhra University. He guided 16 Ph.D.s in the fields of Antennas, Electromagnetics, EMI/EMC and Microwave, Radar Communications, Electronic circuits. Published about 260 technical papers in National/International Journals/Conference Journals and transactions.

He is the recipient of The State Best Teacher Award' from the Government of Andhra Pradesh in 1999, 'The Best Researcher Award' in 1994 ,'Prof. Aiya Memorial National IETE Award' for his best Research guidance in 2008 and Dr. Sarvepalli
Radhakrishnan Award for the Best Academician of the year 2007, He was a visiting Professor in the University of Paderborn and also in the University Karlsruhe, Germany in 1994.

At present he holds the positions of Principal, Andhra University College of Engineering (A), Visakhapatam, Chief Editor of National Journal of Electromagnetic Compatibility.

Prof. Raju has published five textbooks Antennas and Wave Propagation, Electromagnetic Field Theory and Transmission Lines, Electronics Devices and Circuits, Microwave Engineering, Radar Engineering and Navigational Aids

Prof. Raju has been the best faculty performer in Andhra University with the performance index of $99.37 \%$. 